Article

\title{
Platinum-Catalyzed Allylation of 2,3-Disubstituted Indoles with Allylic Acetates
}

\author{
Bai-Jing Peng ${ }^{1}$, Wen-Ting $W^{1}{ }^{1}$ and Shyh-Chyun Yang ${ }^{1,2,3, *}$ \\ 1 School of Pharmacy, College of Pharmacy, Kaohsiung Medical University, Kaohsiung 807, Taiwan; \\ nick4780664@gmail.com (B.-J.P.); jillj6jp6wn6@gmail.com (W.-T.W.) \\ 2 Department of Fragrance and Cosmetic Science, College of Pharmacy, Kaohsiung Medical University, \\ Kaohsiung 807, Taiwan \\ 3 Department of Medical Research, Kaohsiung Medical University Hospital, Kaohsiung 807, Taiwan \\ * Correspondence: scyang@kmu.edu.tw; Tel.:+886-918-665-770
}

Received: 20 October 2017; Accepted: 28 November 2017; Published: 29 November 2017

\begin{abstract}
Given the importance of heterocycle indole derivatives, much effort has been directed toward the development of methods for functionalization of the indole nucleus at $\mathrm{N} 1$ and C3 sites. Moreover, the platinum-catalyzed allyation of nucleophiles was an established and efficient way, which has been applied to medicinal and organic chemistry. In our research, the platinum-catalyzed 2,3-disubstitued indoles with allylic acetates was investigated under different conditions. Herein, we established a simple, convenient, and efficient method, which afforded high yield of allylated indoles.
\end{abstract}

Keywords: platinum-catalyzed; indole derivatives; allylic acetates; allylation

\section{Introduction}

The asymmetric synthesis of indoles is of great interest because of their prevalent structure motifs in natural products, organic materials, and medicinal compounds [1-3]. Especially with indoles and indole-derived heterocycles bearing a 2,3-disubstituted structure in pharmaceutical drugs [4-8]. The 2,3-disubstituted indoles serve as an ambient nucleophile, and some sophisticated conditions are required to achieve selective alkylation either at the $\mathrm{N} 1$ or $\mathrm{C} 3$ position [9-11]. Therefore, given the prevalence of the indole nucleus in biologically active compounds, the direct N1 or C3 functionalization of 2,3-disubstituted indoles represents an important issue [12,13]. Moreover, a particularly difficult transformation is the electrophilic attack at $\mathrm{N} 1$ or $\mathrm{C} 3$ on 2,3-disubstituted indoles to produce indolenines containing a new $\mathrm{C}-\mathrm{C}$ or $\mathrm{C}-\mathrm{N}$ bond [14-16]. For these reasons, extensive efforts have been undertaken to explore the catalytic allylation of indoles at N1 or C3 sites [17-25].

The allylation reaction of transition metal-catalyzed continue to enjoy increasing popularity as these can be based on a variety of metals and demonstrate regioselectivity to branched isomers [26-29]. Transition metal $\eta^{3}$-allyl complexes, as well as transition metal $\sigma$-alkyl complexes, play important roles as active species and key intermediates in many reactions with metal-catalyzed system [30]. The palladium-catalyzed allylation is a powerful tool for $\mathrm{C}-\mathrm{C}, \mathrm{C}-\mathrm{N}$, and $\mathrm{C}-\mathrm{O}$ bond formations, which have been widely applied to organic chemistry [31-36]. The processes have been shown to proceed by attack of nucleophiles on intermediate $\eta^{3}$-allylpalladium (II) complexes generated by oxidative addition of allylic compounds including halides, esters, carbonates, carbamates, phosphates, and related derivatives to a $\operatorname{Pd}(0)$ complex [37-54]. The palladium and ruthenium had been used in the allylation reaction of 2,3-disubstituted indoles, but according to our knowledge, platinum would not be reported [55-57]. The platinum is also a tool for transition metal, which is not often discussed in the reaction of allylation $[58,59]$. In the past course of our studies by using platinum allylation, we established the application of a processed platinum catalysis with satisfied data $[60,61]$. Herein, 
we report a novel catalysis of a platinum complex, which mediates N1-allylation or C3-allylation of 2,3-disubstituted indoles with allylic acetates.

\section{Results and Discussion}

The platinum-catalyzed allylation of 2,3-disubstituted indoles such as 1,2,3,4-tetrahydrocarbazole with allyl acetate was investigated under various conditions (Scheme 1). When a mixture of 1,2,3,4-tetrahydrocarbazole (1a, $1 \mathrm{mmol})$ and allyl acetate (2a, $2 \mathrm{mmol})$ was refluxed in the presence of catalytic amounts of $\mathrm{Pt}(\mathrm{acac})_{2}(2.5 \mathrm{mmol} \%)$ and $\mathrm{PPh}_{3}(10 \mathrm{mmol} \%)$ in benzene for $24 \mathrm{~h}$, $\mathrm{N}$-allyl-1,2,3,4-tetrahydrocarbazole (3a) was formed in only $7 \%$ yield (entry 1 in Table 1). Among the monodentate ligands including $\mathrm{PPh}_{3}$ (entry 1), $\left(2-\mathrm{CH}_{3} \mathrm{C}_{6} \mathrm{H}_{4}\right)_{3} \mathrm{P}$ (entry 2), $\left(3-\mathrm{CH}_{3} \mathrm{C}_{6} \mathrm{H}_{4}\right)_{3} \mathrm{P}$ (entry 3), (4- $\left.\mathrm{CH}_{3} \mathrm{C}_{6} \mathrm{H}_{4}\right)_{3} \mathrm{P}$ (entry 4), (4- $\left.\mathrm{FC}_{6} \mathrm{H}_{4}\right)_{3} \mathrm{P}$ (entry 5), (4- $\left.\mathrm{ClC}_{6} \mathrm{H}_{4}\right)_{3} \mathrm{P}$ (entry 6), (n-butyl) 3 P (entry 7), $\left(3-\mathrm{CH}_{3} \mathrm{OC}_{6} \mathrm{H}_{4}\right)_{3} \mathrm{P}$ (entry 8), $\left(4-\mathrm{CH}_{3} \mathrm{OC}_{6} \mathrm{H}_{4}\right)_{3} \mathrm{P}$ (entry 9), (2-furyl) 3 P (entry 10), (2,6-diCH $\left.\mathrm{H}_{3} \mathrm{OC}_{6} \mathrm{H}_{3}\right)_{3} \mathrm{P}$ (entry 11), and (2,4,6-triC $\left.\mathrm{H}_{3} \mathrm{OC}_{6} \mathrm{H}_{2}\right)_{3} \mathrm{P}$ (entry 12) were used. Furthermore, the bidentate ligands dppm (entry 13), dppf (entry 14), dppe (entry 15), and dppb (entry 16) were evaluated in the reaction. The catalytic reactivity of the ligand $\left(4-\mathrm{ClC}_{6} \mathrm{H}_{4}\right)_{3} \mathrm{P}$ was likely due to improved catalyst stability and got $\mathrm{N}$-allylation product $3 \mathbf{a}$ and $\mathrm{C} 3$-allylation product $4 a$-allyl-2,3,4,4a-tetrahydro- $1 \mathrm{H}$-carbazole (4a) in 69 and 30\% yields, respectively (entry 6). The regisoselectivity N-allylation and C-allylation of 1,2,3,4-tetrahydrocarbazole was about 2:1 ratio. In our condition, $\mathrm{N}$-allylation of 1,2,3,4-tetrahydrocarbazole was the major compound. The predominant $\mathrm{N}$-allylation derivative might be the result of different elements which seemed to control the reaction; however, no decisive conclusion seemed to have been reached. The reaction did not occur in the absence of the phosphine ligand (entry 17) or platinum species (entry 18). The environmental condition was also investigated. At $50{ }^{\circ} \mathrm{C}$, in the presence of $\mathrm{Pt}(\mathrm{acac})_{2}$ and $\left(4-\mathrm{ClC}_{6} \mathrm{H}_{4}\right)_{3} \mathrm{P}$ afforded the yields only $11 \%$ (entry 19$)$. The reaction gave $45 \%$ yields under reflux for $12 \mathrm{~h}$ (entry 20). In the presence of various platinum catalysts, including $\mathrm{Pt}(\text { acac })_{2}$ (entry 6), cis- $\mathrm{PtCl}_{2}(\mathrm{PhCN})_{2}$ (entry 21), cis- $\mathrm{PtCl}_{2}\left(\mathrm{PPh}_{3}\right)_{2}$ (entry 22), di(1,5-cyclooctadiene) Pt (entry 23), $\mathrm{O}\left[\mathrm{Si}\left(\mathrm{CH}_{3}\right)_{2} \mathrm{C}=\mathrm{CH}_{2}\right]_{2} \mathrm{Pt}$ (entry 24), $\mathrm{PtCl}_{2}$ (entry 25), $\mathrm{PtI}_{2}$ (entry 26), $\mathrm{Pt}(\mathrm{CN})_{2}$ (entry 27), $\mathrm{Pt}\left(\mathrm{CH}_{2}=\mathrm{CH}_{2}\right)\left(\mathrm{PPh}_{3}\right)_{2}$ (entries 28 and 29), and $\mathrm{Pt}\left(\mathrm{PPh}_{3}\right)_{4}$ (entries 30 and 31) showed that the most effective platinum catalyst is the $\mathrm{Pt}(\mathrm{acac})_{2}$ (entry 6). However, using $\left.\mathrm{Pt}\left(\mathrm{CH}_{2}=\mathrm{CH}_{2}\right)\left(\mathrm{PPh}_{3}\right)_{2} \mathrm{Or} \mathrm{Pt}_{(\mathrm{PPh}}\right)_{4}$ with extra (4- $\left.\mathrm{ClC}_{6} \mathrm{H}_{4}\right)_{3} \mathrm{P}$ as catalyst increased the yields of products (entries 29 and 31). During the reaction, adding the phosphine ligands could increase the activity of the platinum catalyst. Reduction in the ratio of $\mathrm{Pt}(\mathrm{acac})_{2}$ to $\left(4-\mathrm{ClC}_{6} \mathrm{H}_{4}\right)_{3} \mathrm{P}$ as 1:1 (entry 32), 1:2 (entry 33), and 1:3 (entry 34) ratios decreased the yield in the reaction. It was known that several factors, such as the solvent and nature of the nucleophile, could alter the product pattern in the metal-catalyzed allylation. The six solvents were investigated (entries 6 and 35-39). Although the toxicity of benzene is known, it was the best solvent in this reaction. This survey defined simple and convenient catalyst way for $\mathrm{N}$-allylation and C-allylation of hindered indoles in high yields (entry 6).

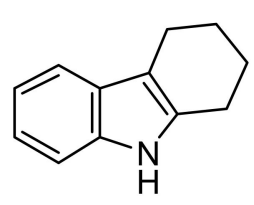

$1 \mathrm{a}$

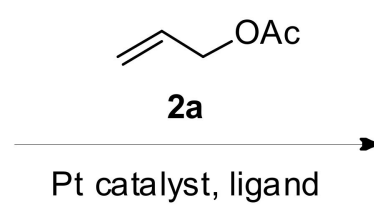

$3 a$
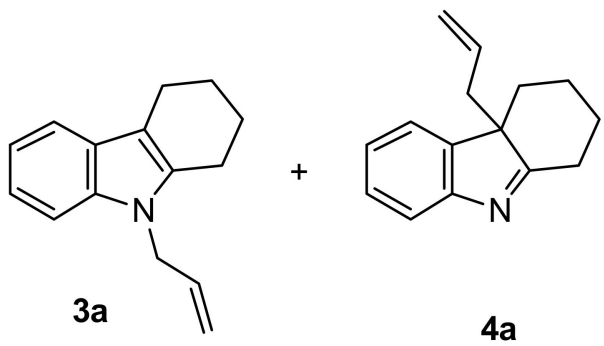

$4 a$

Scheme 1. Allylation of 1,2,3,4-tetrahydrocarbazole (1a) with allyl acetate (2a). 
Table 1. Reaction of 1,2,3,4-tetrahydrocarbazole (1a) with allyl acetate (2a). ${ }^{\text {a }}$

\begin{tabular}{|c|c|c|c|c|}
\hline Entry & Ligand & Platinum Catalyst & Solvent & Yield (\%) (3a:4a) ${ }^{b}$ \\
\hline 1 & $\mathrm{PPh}_{3}$ & $\mathrm{Pt}(\mathrm{acac})_{2}$ & Benzene & $7(7: 0)$ \\
\hline 2 & $\left(2-\mathrm{CH}_{3} \mathrm{C}_{6} \mathrm{H}_{4}\right)_{3} \mathrm{P}$ & $\mathrm{Pt}(\mathrm{acac})_{2}$ & Benzene & $7(0: 7)$ \\
\hline 3 & $\left(3-\mathrm{CH}_{3} \mathrm{C}_{6} \mathrm{H}_{4}\right)_{3} \mathrm{P}$ & $\mathrm{Pt}(\mathrm{acac})_{2}$ & Benzene & $3(0: 3)$ \\
\hline 4 & $\left(4-\mathrm{CH}_{3} \mathrm{C}_{6} \mathrm{H}_{4}\right)_{3} \mathrm{P}$ & $\mathrm{Pt}(\mathrm{acac})_{2}$ & Benzene & $14(8: 6)$ \\
\hline 5 & $\left(4-\mathrm{FC}_{6} \mathrm{H}_{4}\right)_{3} \mathrm{P}$ & $\mathrm{Pt}(\mathrm{acac})_{2}$ & Benzene & $11(0: 11)$ \\
\hline 6 & $\left(4-\mathrm{ClC}_{6} \mathrm{H}_{4}\right)_{3} \mathrm{P}$ & $\mathrm{Pt}(\mathrm{acac})_{2}$ & Benzene & $99(69: 30)$ \\
\hline 7 & (n-butyl) ${ }_{3} \mathrm{P}$ & $\mathrm{Pt}(\mathrm{acac})_{2}$ & Benzene & $30(0: 30)$ \\
\hline 8 & $\left(3-\mathrm{CH}_{3} \mathrm{OC}_{6} \mathrm{H}_{4}\right)_{3} \mathrm{P}$ & $\mathrm{Pt}(\mathrm{acac})_{2}$ & Benzene & $11(3: 8)$ \\
\hline 9 & $\left(4-\mathrm{CH}_{3} \mathrm{OC}_{6} \mathrm{H}_{4}\right)_{3} \mathrm{P}$ & $\mathrm{Pt}(\mathrm{acac})_{2}$ & Benzene & $18(4: 14)$ \\
\hline 10 & (2-furyl $)_{3} \mathrm{P}$ & $\mathrm{Pt}(\mathrm{acac})_{2}$ & Benzene & $8(0: 8)$ \\
\hline 11 & $\left(2,6-\mathrm{diCH}_{3} \mathrm{OC}_{6} \mathrm{H}_{3}\right)_{3} \mathrm{P}$ & $\mathrm{Pt}(\mathrm{acac})_{2}$ & Benzene & $19(9: 10)$ \\
\hline 12 & $\left(2,4,6-\mathrm{triCH}_{3} \mathrm{OC}_{6} \mathrm{H}_{2}\right)_{3} \mathrm{P}$ & $\mathrm{Pt}(\mathrm{acac})_{2}$ & Benzene & $6(0: 6)$ \\
\hline 13 & Dppm $^{\mathrm{c}}$ & $\mathrm{Pt}(\mathrm{acac})_{2}$ & Benzene & $9(5: 4)$ \\
\hline 14 & Dppf $d$ & $\mathrm{Pt}(\mathrm{acac})_{2}$ & Benzene & $13(2: 11)$ \\
\hline 15 & Dppe ${ }^{e}$ & $\mathrm{Pt}(\mathrm{acac})_{2}$ & Benzene & $14(9: 5)$ \\
\hline 16 & $\mathrm{Dppb}^{\mathrm{f}}$ & $\mathrm{Pt}(\mathrm{acac})_{2}$ & Benzene & $12(5: 7)$ \\
\hline 17 & - & $\mathrm{Pt}(\mathrm{acac})_{2}$ & Benzene & $0(0: 0)$ \\
\hline 18 & $\left(4-\mathrm{ClC}_{6} \mathrm{H}_{4}\right)_{3} \mathrm{P}$ & - & Benzene & $0(0: 0)$ \\
\hline $19 \mathrm{~g}$ & $\left(4-\mathrm{ClC}_{6} \mathrm{H}_{4}\right)_{3} \mathrm{P}$ & $\mathrm{Pt}(\mathrm{acac})_{2}$ & Benzene & $11(5: 6)$ \\
\hline $20^{\mathrm{h}}$ & $\left(4-\mathrm{ClC}_{6} \mathrm{H}_{4}\right)_{3} \mathrm{P}$ & $\mathrm{Pt}(\mathrm{acac})_{2}$ & Benzene & $45(32: 13)$ \\
\hline 21 & $\left(4-\mathrm{ClC}_{6} \mathrm{H}_{4}\right)_{3} \mathrm{P}$ & cis- $\mathrm{PtCl}_{2}(\mathrm{PhCN})_{2}$ & Benzene & $16(6: 10)$ \\
\hline 22 & $\left(4-\mathrm{ClC}_{6} \mathrm{H}_{4}\right)_{3} \mathrm{P}$ & cis- $\mathrm{PtCl}_{2}\left(\mathrm{PPh}_{3}\right)_{2}$ & Benzene & $18(5: 13)$ \\
\hline 23 & $\left(4-\mathrm{ClC}_{6} \mathrm{H}_{4}\right)_{3} \mathrm{P}$ & Di(1,5-cyclooctadiene $) \mathrm{Pt}$ & Benzene & $22(7: 15)$ \\
\hline 24 & $\left(4-\mathrm{ClC}_{6} \mathrm{H}_{4}\right)_{3} \mathrm{P}$ & $\mathrm{O}\left[\mathrm{Si}\left(\mathrm{CH}_{3}\right)_{2} \mathrm{C}=\mathrm{CH}_{2}\right]_{2} \mathrm{Pt}$ & Benzene & $12(6: 6)$ \\
\hline 25 & $\left(4-\mathrm{ClC}_{6} \mathrm{H}_{4}\right)_{3} \mathrm{P}$ & $\mathrm{PtCl}_{2}$ & Benzene & $36(11: 25)$ \\
\hline 26 & $\left(4-\mathrm{ClC}_{6} \mathrm{H}_{4}\right)_{3} \mathrm{P}$ & $\mathrm{PtI}_{2}$ & Benzene & $11(0: 11)$ \\
\hline 27 & $\left(4-\mathrm{ClC}_{6} \mathrm{H}_{4}\right)_{3} \mathrm{P}$ & $\operatorname{Pt}(\mathrm{CN})_{2}$ & Benzene & $20(17: 3)$ \\
\hline 28 & - & $\mathrm{Pt}\left(\mathrm{CH}_{2}=\mathrm{CH}_{2}\right)\left(\mathrm{PPh}_{3}\right)_{2}$ & Benzene & $17(2: 15)$ \\
\hline 29 & $\left(4-\mathrm{ClC}_{6} \mathrm{H}_{4}\right)_{3} \mathrm{P}$ & $\mathrm{Pt}\left(\mathrm{CH}_{2}=\mathrm{CH}_{2}\right)\left(\mathrm{PPh}_{3}\right)_{2}$ & Benzene & $35(5: 30)$ \\
\hline 30 & - & $\mathrm{Pt}\left(\mathrm{PPh}_{3}\right)_{4}$ & Benzene & $22(2: 20)$ \\
\hline 31 & $\left(4-\mathrm{ClC}_{6} \mathrm{H}_{4}\right)_{3} \mathrm{P}$ & $\mathrm{Pt}\left(\mathrm{PPh}_{3}\right)_{4}$ & Benzene & $64(4: 60)$ \\
\hline $32^{i}$ & $\left(4-\mathrm{ClC}_{6} \mathrm{H}_{4}\right)_{3} \mathrm{P}$ & $\mathrm{Pt}(\mathrm{acac})_{2}$ & Benzene & $0(0: 0)$ \\
\hline $33^{j}$ & $\left(4-\mathrm{ClC}_{6} \mathrm{H}_{4}\right)_{3} \mathrm{P}$ & $\mathrm{Pt}(\mathrm{acac})_{2}$ & Benzene & $11(5: 6)$ \\
\hline $34^{k}$ & $\left(4-\mathrm{ClC}_{6} \mathrm{H}_{4}\right)_{3} \mathrm{P}$ & $\mathrm{Pt}(\mathrm{acac})_{2}$ & Benzene & $75(38: 37)$ \\
\hline 35 & $\left(4-\mathrm{ClC}_{6} \mathrm{H}_{4}\right)_{3} \mathrm{P}$ & $\mathrm{Pt}(\mathrm{acac})_{2}$ & Toluene & $82(62: 20)$ \\
\hline 36 & $\left(4-\mathrm{ClC}_{6} \mathrm{H}_{4}\right)_{3} \mathrm{P}$ & $\mathrm{Pt}(\mathrm{acac})_{2}$ & $\mathrm{CH}_{2} \mathrm{Cl}_{2}$ & $43(40: 3)$ \\
\hline 37 & $\left(4-\mathrm{ClC}_{6} \mathrm{H}_{4}\right)_{3} \mathrm{P}$ & $\mathrm{Pt}(\mathrm{acac})_{2}$ & THF & $52(34: 18)$ \\
\hline 38 & $\left(4-\mathrm{ClC}_{6} \mathrm{H}_{4}\right)_{3} \mathrm{P}$ & $\mathrm{Pt}(\mathrm{acac})_{2}$ & Dioxane & $78(63: 15)$ \\
\hline 39 & $\left(4-\mathrm{ClC}_{6} \mathrm{H}_{4}\right)_{3} \mathrm{P}$ & $\mathrm{Pt}(\mathrm{acac})_{2}$ & DMF & $67(53: 14)$ \\
\hline
\end{tabular}

\footnotetext{
a Reaction conditions: 1a $(1 \mathrm{mmol}), 2 \mathrm{a}(2 \mathrm{mmol}), \mathrm{Pt}$ catalyst $(0.025 \mathrm{mmol})$, and ligand $(0.1 \mathrm{mmol})$ in solvent $(5 \mathrm{~mL})$ were refluxed for $24 \mathrm{~h}$. b Isolated yield. c 1,1-Bis(diphenylphosphino)methane. $\mathrm{d}$ 1,1-Bis(diphenylphosphino)ferrocene. ${ }^{\mathrm{e}}$ 1,2-Bis(diphenylphosphino)ethane. ${ }^{\mathrm{f}}$ 1,4-Bis(diphenylphosphino)butane. g Stirred at $50{ }^{\circ} \mathrm{C} .{ }^{\mathrm{h}}$ Refluxed for $12 \mathrm{~h} .{ }^{\mathrm{i}}\left(4-\mathrm{ClC}_{6} \mathrm{H}_{4}\right)_{3} \mathrm{P}(0.025 \mathrm{mmol}) .{ }^{\mathrm{j}}\left(4-\mathrm{ClC}_{6} \mathrm{H}_{4}\right)_{3} \mathrm{P}(0.05 \mathrm{mmol}) .{ }^{\mathrm{k}}\left(4-\mathrm{ClC}_{6} \mathrm{H}_{4}\right)_{3} \mathrm{P}$ $(0.075 \mathrm{mmol})$.
}

The high efficiency of the allylation reactions described above encouraged us to extend the reaction to allylic compounds. The results for allylation of a number of allylic compounds $\mathbf{2} \mathbf{b}-\mathbf{f}$ with 1,2,3,4-tetrahydrocarbazole (1a) using $\mathrm{Pt}(\mathrm{acac})_{2}$ and $\left(4-\mathrm{ClC}_{6} \mathrm{H}_{4}\right)_{3} \mathrm{P}$ were summarized in Table 2. 3-Buten-2-yl acetate (2b) which reacted with $1 \mathbf{a}$ gave $3 \mathbf{b}$ and $4 \mathbf{b}$ in 54 and $23 \%$ yields, respectively (entry 1 ). The $E / Z$ ratio of $\mathbf{3 b}$ and $\mathbf{4} \mathbf{b}$ was determined by GC. The product $E$ alkene generated from the more thermodynamically stable syn complex. The corresponding reaction with crotyl acetate (2c) afforded $\mathrm{N}$-allylated and C-allylated tetrahydrocarbazole in overall $87 \%$ yields (entry 2). These products might all be derived from the same $\pi$-allylic intermediate which could be attacked at the $\mathrm{C}-1$. The reaction was considered to proceed via $\pi$-allylplatinum intermediates. The loss of stereochemistry of starting acetate $\mathbf{2 b}$ was due to a rapid $\sigma \leftrightarrow \eta^{3} \leftrightarrow \sigma$ interconversion of the intermediates 
compared to the rate of allylation. Allylation of trans-2-hexen-1-yl acetate (2d) gave mixtures of $\mathrm{N}$-allylated and C-allylated tetrahydrocarbazole 3c and $4 \mathrm{c}$ in yields of 45 and 27\%, respectively (entry 3). Reaction of allyl chloride (2e) produced 3a and $\mathbf{4 a}$ in yields of 34 and 12\%, respectively (entry 4). The allyl chloride is not a good reagent for allylation, but with $\mathrm{Pt}(\mathrm{acac})_{2}(0.05 \mathrm{mmol})$ and $\left(4-\mathrm{ClC}_{6} \mathrm{H}_{4}\right)_{3} \mathrm{P}(0.2 \mathrm{mmol})$, the totally yields increased to $75 \%$ (entry 5$)$. Lastly, reaction of $1 \mathrm{a}$ with allyl carbonate (2f) afforded $3 \mathbf{a}$ and $\mathbf{4 a}$ in overall $92 \%$ yields (entry 6 ).

Table 2. Reaction of 1,2,3,4-tetrahydrocarbazole (1a) with allylic compounds (2b-f). ${ }^{\text {a }}$

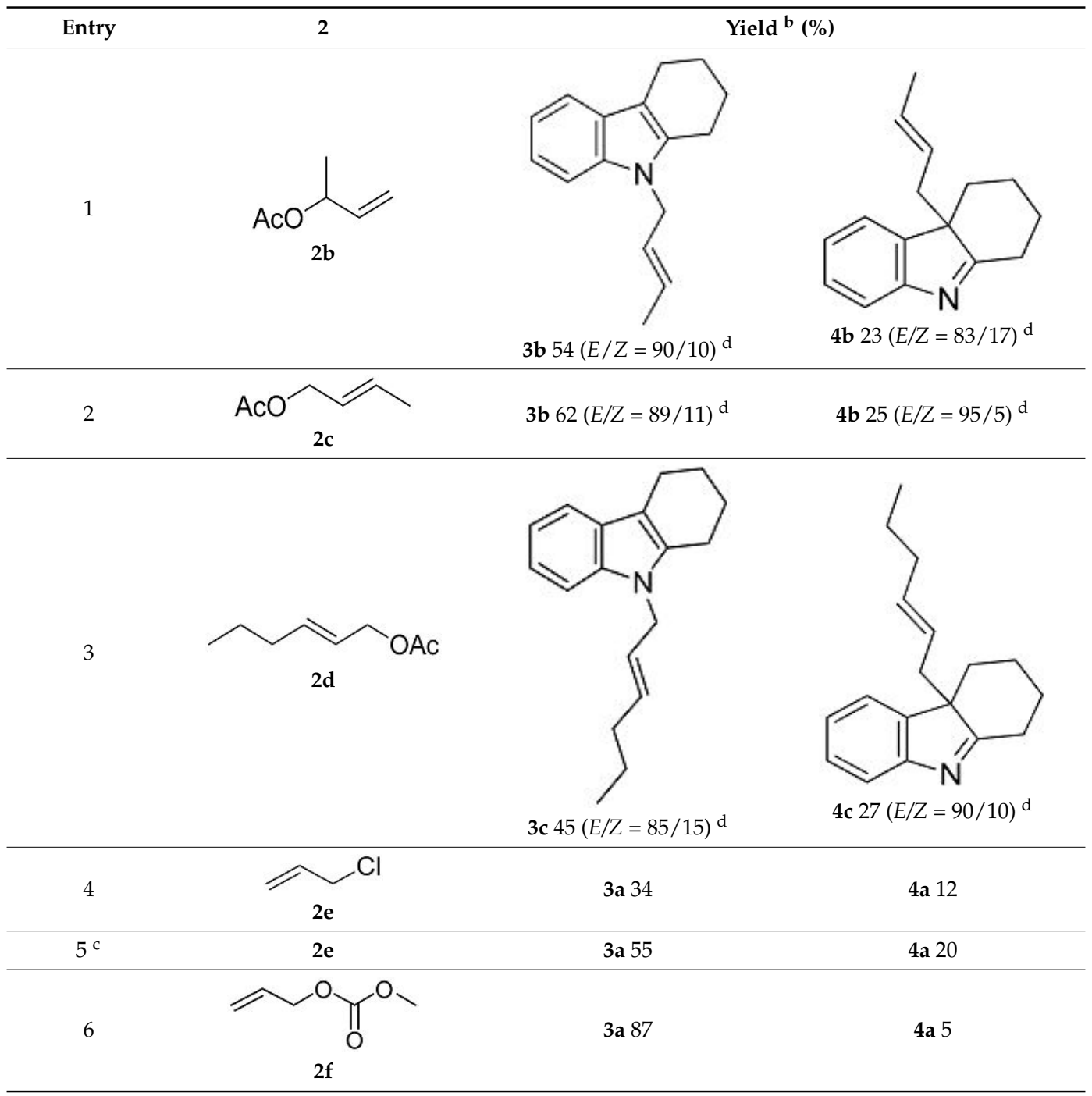

a Reaction conditions: 1a $(1 \mathrm{mmol}), 2(2 \mathrm{mmol}), \mathrm{Pt}(\mathrm{acac})_{2}(0.025 \mathrm{mmol})$, and $\left(4-\mathrm{ClC}_{6} \mathrm{H}_{4}\right)_{3} \mathrm{P}(0.1 \mathrm{mmol})$ in benzene $(5 \mathrm{~mL})$ were refluxed for $24 \mathrm{~h} .{ }^{\mathrm{b}}$ Isolated yield. ${ }^{\mathrm{c}} \mathrm{Pt}(\mathrm{acac})_{2}(0.05 \mathrm{mmol})$ and $\left(4-\mathrm{ClC}_{6} \mathrm{H}_{4}\right)_{3} \mathrm{P}(0.2 \mathrm{mmol}) .{ }^{\mathrm{d}}$ Determined by GC.

The reaction conditions developed above were found to be useful and efficient to corresponding indole derivatives (Table 3). The results collected in Table 3 shown that allylation of allyl acetate (2a) with indoles using $\mathrm{Pt}(\mathrm{acac})_{2}$ and $\left(4-\mathrm{ClC}_{6} \mathrm{H}_{4}\right)_{3} \mathrm{P}$, giving general good yields of the corresponding allylic indoles (entries 1-3). Cycloheptane-fused indole (1b) was used in the reaction and gave $97 \%$ yields of the corresponding $\mathrm{N}$-allylated and C-allylated products (entry 1). 2,3-Dimethyl indole (1c) was under investigation. The overall yield was $98 \%$ (entry 2). Finally, allylation of the simpler 3-methylindole (1d) was tested in the reaction. 3-Methylindole (1d) generated the allylation products $3 f$ in a $47 \%$ yield and $4 \mathrm{f}$ in a $24 \%$ yield after $24 \mathrm{~h}$ (entry 3$)$. 
Table 3. Allylation of indoles (1) with allyl acetate (2a). ${ }^{\text {a }}$

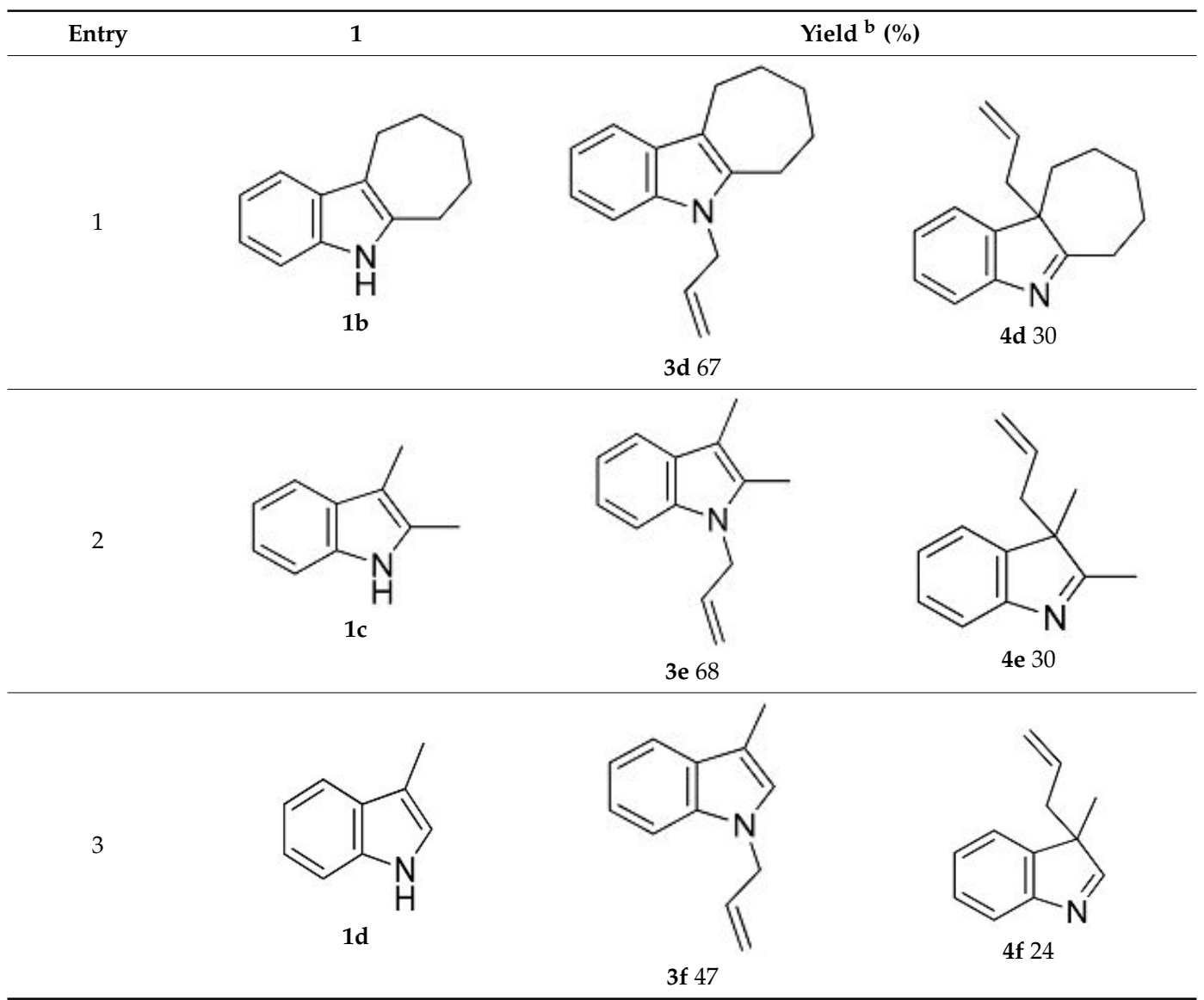

a Reaction conditions: $\mathbf{1}(1 \mathrm{mmol}), \mathbf{2 a}(2 \mathrm{mmol}), \mathrm{Pt}(\mathrm{acac})_{2}(0.025 \mathrm{mmol})$, and $\left(4-\mathrm{ClC}_{6} \mathrm{H}_{4}\right)_{3} \mathrm{P}(0.1 \mathrm{mmol})$ in benzene $(5 \mathrm{~mL})$ were refluxed for $24 \mathrm{~h} .{ }^{\mathrm{b}}$ Isolated yield.

\section{Experimental Section}

\subsection{General Considerations}

Reagents were obtained from Acros Organics (Geel, Belgium), Tokyo Chemical Industry (Tokyo, Japan), Sigma-Aldrich (St. Louis, MO, USA), and Alfa-Aesar (Ward Hill, MA, USA), and used without further purification. All reactions were carried out under a nitrogen atmosphere. Solvents were dried and distilled by known methods. Cloumn chromatography was performed on silica gel. IR absorption spectra were recorded on Shimadzu IR-27G and Perkin-Elmer System 2000FT-IR spectrophotometers. Proton nuclear magnetic resonance $\left({ }^{1} \mathrm{H}-\mathrm{NMR}, 400 \mathrm{MHz}\right)$ and carbon-13 NMR spectra were measured with Varian Unity-400 spectrometers. Carbon multiplicities were obtained from DEPT experiments. Chemical shifts $(\delta)$ and coupling constants $(\mathrm{Hz})$ were measured with respect to TMS or chloroform- $d_{1}$. Mass and high-resolution mass spectra (HRMS) were taken on a Hewlett-Packard 5989A or JEOL JMS D-100 instrument, with a direct inlet system.

\subsection{General Procedure}

A mixture of 1,2,3,4-tetrahydrocarbazole (1a, $1 \mathrm{mmol})$, allyl acetate (2a, $2 \mathrm{mmol}), \mathrm{Pt}(\mathrm{acac})_{2}$ $(9.7 \mathrm{mg}, 0.025 \mathrm{mmol})$, and $\left(4-\mathrm{ClC}_{6} \mathrm{H}_{4}\right)_{3} \mathrm{P}(36.5 \mathrm{mg}, 0.1 \mathrm{mmol})$ in benzene $(5 \mathrm{~mL})$ was refluxed for $24 \mathrm{~h}$. After cooling, the solvent was distilled under reduced pressure. Column chromatography $(n$-hexane $/$ EtOAc $=4: 1)$ of the residue afforded $146 \mathrm{mg}(69 \%)$ of $3 \mathbf{a}$ and $63 \mathrm{mg}(30 \%) 4 \mathbf{a}$, respectively. 
$0.8 \mathrm{~Hz}, 1 \mathrm{H}, \mathrm{ArH}), 7.06(\mathrm{ddd}, J=8.4,6.8,0.8 \mathrm{~Hz}, 1 \mathrm{H}, \mathrm{ArH}), 5.90(\mathrm{ddt}, J=17.2,10.4,4.8 \mathrm{~Hz}, 1 \mathrm{H}$, vinyl H), 5.09 (ddt, $J=10.4,1.6,1.6 \mathrm{~Hz}, 1 \mathrm{H}$, vinyl H), $4.88(\mathrm{ddt}, J=17.2,1.6,1.6 \mathrm{~Hz}, 1 \mathrm{H}$, vinyl $\mathrm{H}), 4.62(\mathrm{dt}, J=4.8$, $\left.1.6 \mathrm{~Hz}, 2 \mathrm{H}, \mathrm{NCH}_{2}\right), 2.73\left(\mathrm{tt}, J=6.0,1.6 \mathrm{~Hz}, 2 \mathrm{H}, \mathrm{CH}_{2}\right), 2.67\left(\mathrm{tt}, J=6.0,1.6 \mathrm{~Hz}, 2 \mathrm{H}, \mathrm{CH}_{2}\right), 1.82-1.96(\mathrm{~m}$, $\left.4 \mathrm{H}, \mathrm{CH}_{2} \times 2\right) ;{ }^{13} \mathrm{C}-\mathrm{NMR}\left(100 \mathrm{MHz}, \mathrm{CDCl}_{3}\right): \delta 136.2(\mathrm{C}), 135.4(\mathrm{C}), 133.8(\mathrm{CH}), 127.4(\mathrm{C}), 120.5(\mathrm{CH})$, $118.7(\mathrm{CH}), 117.7(\mathrm{CH}), 116.0\left(\mathrm{CH}_{2}\right), 109.5(\mathrm{C}), 108.8(\mathrm{CH}), 45.0\left(\mathrm{CH}_{2}\right), 23.2\left(\mathrm{CH}_{2}\right), 23.2\left(\mathrm{CH}_{2}\right), 22.0$ $\left(\mathrm{CH}_{2}\right), 21.1\left(\mathrm{CH}_{2}\right)$. EI-MS: $m / z 211\left(\mathrm{M}^{+}\right), 196,183,168,154,142,128,115,89,77,63,51$. EI-HRMS calcd. for $\mathrm{C}_{15} \mathrm{H}_{17} \mathrm{~N}$ : 211.1361. Found: 211.1363.

4a-Allyl-2,3,4,4a-tetrahydro-1H-carbazole (4a) [62]: yellow oil. IR (KBr): $v 1711,1641,1617,1451 \mathrm{~cm}^{-1}$. ${ }^{1} \mathrm{H}-\mathrm{NMR}\left(400 \mathrm{MHz}, \mathrm{CDCl}_{3}\right): \delta 7.58(\mathrm{~d}, J=7.6 \mathrm{~Hz}, 1 \mathrm{H}, \mathrm{ArH}), 7.31(\mathrm{ddd}, J=7.6,7.2,1.2 \mathrm{~Hz}, 1 \mathrm{H}, \mathrm{ArH})$, $7.30(\mathrm{dd}, J=7.6,1.2 \mathrm{~Hz}, 1 \mathrm{H}, \mathrm{ArH}), 7.17(\mathrm{ddd}, J=7.6,7.2,1.2 \mathrm{~Hz}, 1 \mathrm{H}, \mathrm{ArH}), 5.17(\mathrm{ddt}, J=17.2,10.0$, $6.8 \mathrm{~Hz}, 1 \mathrm{H}$, vinyl H), $4.94(\mathrm{ddt}, J=17.2,2.0,1.2 \mathrm{~Hz}, 1 \mathrm{H}$, vinyl $\mathrm{H}), 4.86(\mathrm{ddt}, J=10.0,2.0,1.2 \mathrm{~Hz}, 1 \mathrm{H}$, vinyl H), 2.85-2.91 (m, 1H, CH), $2.62(\mathrm{dt}, J=13.2,6.4 \mathrm{~Hz}, 1 \mathrm{H}, \mathrm{CH}), 2.54-2.59(\mathrm{~m}, 1 \mathrm{H}, \mathrm{CH}), 2.54(\mathrm{dt}, J=13.2$, $5.6 \mathrm{~Hz}, 1 \mathrm{H}, \mathrm{CH}) 2.36(\mathrm{ddt}, J=13.2,3.2,2.8 \mathrm{~Hz}, 1 \mathrm{H}, \mathrm{CH}), 2.18-2.24(\mathrm{~m}, 1 \mathrm{H}, \mathrm{CH}), 1.83(\mathrm{tq}, J=13.6,4.0 \mathrm{~Hz}$, $1 \mathrm{H}, \mathrm{CH}), 1.66-1.72(\mathrm{~m}, 1 \mathrm{H}, \mathrm{CH}), 1.43(\mathrm{tq}, J=13.6,4.0 \mathrm{~Hz}, 1 \mathrm{H}, \mathrm{CH}), 1.16(\mathrm{dt}, J=13.6,4.0 \mathrm{~Hz}, 1 \mathrm{H}, \mathrm{CH})$; ${ }^{13} \mathrm{C}-\mathrm{NMR}\left(100 \mathrm{MHz}, \mathrm{CDCl}_{3}\right): \delta 188.9(\mathrm{C}), 154.8(\mathrm{C}), 144.6(\mathrm{C}), 132.1(\mathrm{CH}), 127.6(\mathrm{CH}), 124.7(\mathrm{CH}), 121.9$ $(\mathrm{CH}), 120.1(\mathrm{CH}), 118.0\left(\mathrm{CH}_{2}\right), 57.6(\mathrm{C}), 37.6\left(\mathrm{CH}_{2}\right), 37.0\left(\mathrm{CH}_{2}\right), 30.1\left(\mathrm{CH}_{2}\right), 28.8\left(\mathrm{CH}_{2}\right), 21.1\left(\mathrm{CH}_{2}\right)$. EI-MS: $m / z 211\left(\mathrm{M}^{+}\right), 196,183,170,168,154,142,128,115,89,77,63,51$. EI-HRMS calcd. for $\mathrm{C}_{15} \mathrm{H}_{17} \mathrm{~N}$ : 211.1361. Found: 211.1358.

N-(But-2-en-1-yl)-1,2,3,4-tetrahydrocarbazole (3b): yellow oil. IR (KBr): $v 1653,1611,1462 \mathrm{~cm}^{-1} .{ }^{1} \mathrm{H}-\mathrm{NMR}$ $(400 \mathrm{MHz}, \mathrm{CDCl} 3): \delta 7.46(\mathrm{dd}, J=8.0,1.2 \mathrm{~Hz}, 1 \mathrm{H}, \mathrm{ArH}), 7.24(\mathrm{dd}, J=8.0,1.2 \mathrm{~Hz}, 1 \mathrm{H}, \mathrm{ArH}), 7.12$ (ddd, $J=8.0,7.2,1.2 \mathrm{~Hz}, 1 \mathrm{H}, \mathrm{ArH}), 7.05(\mathrm{ddd}, J=8.0,7.2,1.2 \mathrm{~Hz}, 1 \mathrm{H}, \mathrm{ArH}), 5.40-5.61(\mathrm{~m}, 2 \mathrm{H}, \operatorname{vinyl~H})$, $4.53-4.55\left(\mathrm{~m}, 2 \mathrm{H}, \mathrm{NCH}_{2}\right), 2.73\left(\mathrm{tt}, J=6.0,1.6 \mathrm{~Hz}, 2 \mathrm{H}, \mathrm{CH}_{2}\right), 2.68\left(\mathrm{tt}, J=6.0,1.6 \mathrm{~Hz}, 2 \mathrm{H}, \mathrm{CH}_{2}\right), 1.80-1.95$ $\left(\mathrm{m}, 4 \mathrm{H}, \mathrm{CH}_{2} \times 2\right), 1.63\left(\mathrm{dd}, J=6.4,1.2 \mathrm{~Hz}, 3 \mathrm{H}, \mathrm{CH}_{3}\right) ;{ }^{13} \mathrm{C}-\mathrm{NMR}\left(100 \mathrm{MHz}, \mathrm{CDCl}_{3}\right): \delta 136.0(\mathrm{C}), 135.3$ (C), $127.3(\mathrm{C}), 126.7(\mathrm{CH}), 120.4(\mathrm{CH}), 118.6(\mathrm{CH}), 117.7(\mathrm{CH}), 109.3(\mathrm{C}), 108.9(\mathrm{CH}), 44.4\left(\mathrm{CH}_{2}\right), 23.3$ $\left(\mathrm{CH}_{2}\right), 23.2\left(\mathrm{CH}_{2}\right), 22.1\left(\mathrm{CH}_{2}\right), 21.1\left(\mathrm{CH}_{2}\right), 17.5\left(\mathrm{CH}_{3}\right)$. EI-MS: $\mathrm{m} / z 225\left(\mathrm{M}^{+}\right), 210,197,182,168,154,143$, $128,115,89,77,63,51$. EI-HRMS calcd. for $\mathrm{C}_{16} \mathrm{H}_{19} \mathrm{~N}$ : 225.1517. Found: 225.1517.

4a-(But-2-en-1-yl)-2,3,4,4a-tetrahydro-1H-carbazole (4b) [16]: yellow oil. IR (KBr): $v$ 1711, 1616, 1584, $1450 \mathrm{~cm}^{-1} .{ }^{1} \mathrm{H}-\mathrm{NMR}\left(400 \mathrm{MHz}, \mathrm{CDCl}_{3}\right): \delta 7.57(\mathrm{~d}, J=7.6 \mathrm{~Hz}, 1 \mathrm{H}, \mathrm{ArH}), 7.26-7.34(\mathrm{~m}, 2 \mathrm{H}, \mathrm{ArH}), 7.17$ $(\mathrm{t}, J=7.6 \mathrm{~Hz}, 1 \mathrm{H}, \mathrm{ArH}), 5.37(\mathrm{dq}, J=13.6,6.4 \mathrm{~Hz}, 1 \mathrm{H}$, vinyl $\mathrm{H}), 4.83(\mathrm{tq}, J=13.6,6.4 \mathrm{~Hz}, 1 \mathrm{H}$, vinyl $\mathrm{H}), 2.84-2.89(\mathrm{~m}, 1 \mathrm{H}, \mathrm{CH}), 2.55-2.61(\mathrm{~m}, 1 \mathrm{H}, \mathrm{CH}) 2.54(\mathrm{dt}, J=13.6,5.6 \mathrm{~Hz}, 1 \mathrm{H}, \mathrm{CH}) 2.44(\mathrm{dd}, J=13.6$, $7.6 \mathrm{~Hz}, 1 \mathrm{H}, \mathrm{CH}) 2.34(\mathrm{dq}, J=13.6,2.8 \mathrm{~Hz}, 1 \mathrm{H}, \mathrm{CH}), 2.15-2.24(\mathrm{~m}, 1 \mathrm{H}, \mathrm{CH}), 1.81(\mathrm{tq}, J=13.6,4.0 \mathrm{~Hz}, 1 \mathrm{H}$, $\mathrm{CH}), 1.64-1.69(\mathrm{~m}, 1 \mathrm{H}, \mathrm{CH}), 1.48\left(\mathrm{dd}, J=6.4,0.8 \mathrm{~Hz}, 3 \mathrm{H}, \mathrm{CH}_{3}\right), 1.41(\mathrm{tq}, J=13.6,4.0 \mathrm{~Hz}, 1 \mathrm{H}, \mathrm{CH}), 1.12$ $(\mathrm{dt}, J=13.6,4.0 \mathrm{~Hz}, 1 \mathrm{H}, \mathrm{CH}) ;{ }^{13} \mathrm{C}-\mathrm{NMR}\left(100 \mathrm{MHz}, \mathrm{CDCl}_{3}\right): \delta 189.2(\mathrm{C}), 154.8(\mathrm{C}), 144.9(\mathrm{C}), 128.6(\mathrm{CH})$, $127.4(\mathrm{CH}), 124.5(\mathrm{CH}), 124.4(\mathrm{CH}), 121.9(\mathrm{CH}), 120.0(\mathrm{CH}), 57.8(\mathrm{C}), 36.7\left(\mathrm{CH}_{2}\right), 36.4\left(\mathrm{CH}_{2}\right), 30.1\left(\mathrm{CH}_{2}\right)$, $28.8\left(\mathrm{CH}_{2}\right), 21.0\left(\mathrm{CH}_{2}\right), 17.7\left(\mathrm{CH}_{3}\right)$. EI-MS: $m / z 225\left(\mathrm{M}^{+}\right), 210,196,182,168,154,143,128,115,89,77,63$. EI-HRMS calcd. for $\mathrm{C}_{16} \mathrm{H}_{19} \mathrm{~N}$ : 225.1517. Found: 225.1518.

$\mathrm{N}$-(Hex-2-en-1-yl)-1,2,3,4-tetrahydrocarbazole (3c): yellow oil. IR (KBr): $v 1658,1613,1464 \mathrm{~cm}^{-1} .{ }^{1} \mathrm{H}-\mathrm{NMR}$ $\left(400 \mathrm{MHz}, \mathrm{CDCl}_{3}\right): \delta 7.54(\mathrm{~d}, J=7.6 \mathrm{~Hz}, 1 \mathrm{H}, \mathrm{ArH}), 7.32(\mathrm{~d}, J=8.0 \mathrm{~Hz}, 1 \mathrm{H}, \mathrm{ArH}), 7.20(\mathrm{dt}, J=8.0,1.2 \mathrm{~Hz}$, $1 \mathrm{H}, \mathrm{ArH}), 7.13(\mathrm{dt}, J=7.6,1.2 \mathrm{~Hz}, 1 \mathrm{H}, \operatorname{ArH}), 5.47-5.61(\mathrm{~m}, 2 \mathrm{H}$, vinyl H), $4.63(\mathrm{dd}, J=4.8,1.2 \mathrm{~Hz}, 2 \mathrm{H}$, $\left.\mathrm{NCH}_{2}\right), 2.81\left(\mathrm{t}, J=6.0 \mathrm{~Hz}, 2 \mathrm{H}, \mathrm{CH}_{2}\right), 2.75\left(\mathrm{t}, J=6.0 \mathrm{~Hz}, 2 \mathrm{H}, \mathrm{CH}_{2}\right), 1.90-2.05\left(\mathrm{~m}, 6 \mathrm{H}, \mathrm{CH}_{2} \times 3\right), 1.41$ (hext, $\left.J=7.6 \mathrm{~Hz}, 2 \mathrm{H}, \mathrm{CH}_{2}\right), 0.92\left(\mathrm{t}, J=7.6 \mathrm{~Hz}, 3 \mathrm{H}, \mathrm{CH}_{3}\right) ;{ }^{13} \mathrm{C}-\mathrm{NMR}\left(100 \mathrm{MHz}, \mathrm{CDCl}_{3}\right): \delta 136.1(\mathrm{C})$, $135.3(\mathrm{C}), 132.6(\mathrm{CH}), 127.3(\mathrm{C}), 125.6(\mathrm{CH}), 120.4(\mathrm{CH}), 118.5(\mathrm{CH}), 117.6(\mathrm{CH}), 109.3(\mathrm{C}), 108.9(\mathrm{CH})$, 44.6 $\left(\mathrm{CH}_{2}\right), 36.5\left(\mathrm{CH}_{2}\right), 23.3\left(\mathrm{CH}_{2}\right), 23.2\left(\mathrm{CH}_{2}\right), 22.2\left(\mathrm{CH}_{2}\right), 22.1\left(\mathrm{CH}_{2}\right), 21.1\left(\mathrm{CH}_{2}\right), 13.6\left(\mathrm{CH}_{3}\right)$. EI-MS: $\mathrm{m} / \mathrm{z} 253\left(\mathrm{M}^{+}\right), 225,210,196,182,168,154,143,128,115,89,77,63,55$. EI-HRMS calcd. for $\mathrm{C}_{18} \mathrm{H}_{23} \mathrm{~N}$ : 253.1830. Found: 253.1830.

4a-(Hex-2-en-1-yl)-2,3,4,4a-tetrahydro-1H-carbazole (4c): yellow oil. IR (KBr): $v$ 1711, 1616, 1586, $1463 \mathrm{~cm}^{-1} .{ }^{1} \mathrm{H}-\mathrm{NMR}\left(400 \mathrm{MHz}, \mathrm{CDCl}_{3}\right): \delta 7.57(\mathrm{~d}, J=7.6 \mathrm{~Hz}, 1 \mathrm{H}, \mathrm{ArH}), 7.31(\mathrm{dt}, J=7.6,1.2 \mathrm{~Hz}$, $1 \mathrm{H}, \mathrm{ArH}), 7.28(\mathrm{dd}, J=7.6,1.2 \mathrm{~Hz}, 1 \mathrm{H}, \mathrm{ArH}), 7.17(\mathrm{dt}, J=7.6,1.2 \mathrm{~Hz}, 1 \mathrm{H}, \mathrm{ArH}), 5.33(\mathrm{dt}, J=15.2,6.8 \mathrm{~Hz}$, 
$1 \mathrm{H}$, vinyl $\mathrm{H}), 4.81(\mathrm{dt}, J=15.2,6.8 \mathrm{~Hz}, 1 \mathrm{H}$, vinyl $\mathrm{H}), 2.85-2.90(\mathrm{~m}, 1 \mathrm{H}, \mathrm{CH}), 2.53-2.59\left(\mathrm{~m}, 2 \mathrm{H}, \mathrm{CH}_{2}\right), 2.49$ $(\mathrm{dt}, J=13.6,7.6 \mathrm{~Hz}, 1 \mathrm{H}, \mathrm{CH}), 2.35(\mathrm{dq}, J=13.6,2.8 \mathrm{~Hz}, 1 \mathrm{H}, \mathrm{CH}), 2.16-2.23(\mathrm{~m}, 1 \mathrm{H}, \mathrm{CH}), 1.65-1.88(\mathrm{~m}$, $4 \mathrm{H}, \mathrm{CH} \times 4), 1.43(\mathrm{tq}, J=13.6,4.4 \mathrm{~Hz}, 1 \mathrm{H}, \mathrm{CH}), 1.15-1.23\left(\mathrm{~m}, 2 \mathrm{H}, \mathrm{CH}_{2}\right), 1.14(\mathrm{dt}, J=13.6,4.4 \mathrm{~Hz}, 1 \mathrm{H}$, $\mathrm{CH}), 0.73\left(\mathrm{t}, J=7.2, \mathrm{~Hz}, 3 \mathrm{H}, \mathrm{CH}_{3}\right) ;{ }^{13} \mathrm{C}-\mathrm{NMR}\left(100 \mathrm{MHz}, \mathrm{CDCl}_{3}\right): \delta 189.2(\mathrm{C}), 154.8(\mathrm{C}), 144.9$ (C), 134.2 $(\mathrm{CH}), 127.4(\mathrm{CH}), 124.5(\mathrm{CH}), 123.3(\mathrm{CH}), 121.9(\mathrm{CH}), 120.0(\mathrm{CH}), 57.9(\mathrm{C}), 36.7\left(\mathrm{CH}_{2}\right), 36.4\left(\mathrm{CH}_{2}\right), 34.3$ $\left(\mathrm{CH}_{2}\right), 30.1\left(\mathrm{CH}_{2}\right), 28.8\left(\mathrm{CH}_{2}\right), 22.4\left(\mathrm{CH}_{2}\right) 21.1\left(\mathrm{CH}_{2}\right), 13.4\left(\mathrm{CH}_{3}\right)$. EI-MS: $m / z 253\left(\mathrm{M}^{+}\right), 225,210,196$, $182,168,154,143,128,115,89,77,63,55$. EI-HRMS calcd. for $\mathrm{C}_{18} \mathrm{H}_{23} \mathrm{~N}$ : 253.1830. Found: 253.1832.

N-Allyl-6,7,8,9,10,10a-hexahydro-cyclohepta[b]indole (3d): yellow oil. IR (KBr): $v 1643,1611,1465 \mathrm{~cm}^{-1}$. ${ }^{1} \mathrm{H}-\mathrm{NMR}\left(400 \mathrm{MHz}, \mathrm{CDCl}_{3}\right): \delta 7.56(\mathrm{dd}, J=7.6 \mathrm{~Hz}, 1.2,1 \mathrm{H}, \mathrm{ArH}), 7.25(\mathrm{dd}, J=7.2,1.2 \mathrm{~Hz}, 1 \mathrm{H}, \mathrm{ArH})$, $7.17(\mathrm{dt}, J=7.2,1.2 \mathrm{~Hz}, 1 \mathrm{H}, \mathrm{ArH}), 7.13(\mathrm{dt}, J=7.2,1.2 \mathrm{~Hz}, 1 \mathrm{H}, \mathrm{ArH}), 5.97(\mathrm{ddt}, J=17.2,10.4,4.4 \mathrm{~Hz}$, $1 \mathrm{H}$, vinyl $\mathrm{H}), 5.14(\mathrm{ddt}, J=10.4,2.0,1.2 \mathrm{~Hz}, 1 \mathrm{H}$, vinyl $\mathrm{H}), 4.88(\mathrm{ddt}, J=17.2,2.0,1.2 \mathrm{~Hz}, 1 \mathrm{H}$, vinyl $\mathrm{H})$, $4.73\left(\mathrm{dt}, J=4.4,2.0 \mathrm{~Hz}, 2 \mathrm{H}, \mathrm{NCH}_{2}\right), 2.84-2.93\left(\mathrm{~m}, 4 \mathrm{H}, \mathrm{CH}_{2} \times 2\right), 1.80-1.98\left(\mathrm{~m}, 6 \mathrm{H}, \mathrm{CH}_{2} \times 3\right) ;{ }^{13} \mathrm{C}-\mathrm{NMR}$ $\left(100 \mathrm{MHz}, \mathrm{CDCl}_{3}\right): \delta 138.8(\mathrm{C}), 135.3(\mathrm{C}), 134.0(\mathrm{CH}), 127.9(\mathrm{C}), 120.3(\mathrm{CH}), 118.7(\mathrm{CH}), 117.6(\mathrm{CH})$, 115.8 $\left(\mathrm{CH}_{2}\right), 113.9(\mathrm{C}), 108.8(\mathrm{CH}), 45.0\left(\mathrm{CH}_{2}\right), 31.7\left(\mathrm{CH}_{2}\right), 28.4\left(\mathrm{CH}_{2}\right), 27.2\left(\mathrm{CH}_{2}\right), 26.3\left(\mathrm{CH}_{2}\right) 24.4\left(\mathrm{CH}_{2}\right)$. EI-MS: $m / z 225\left(\mathrm{M}^{+}\right), 209,196,182,168,156,142,128,115,89,77,63,51$. EI-HRMS calcd. for $\mathrm{C}_{16} \mathrm{H}_{19} \mathrm{~N}$ : 225.1517. Found: 225.1517.

10a-Allyl-6,7,8,9,10,10a-hexahydrocyclohepta[b]indole (4d) [16]: yellow oil. IR (KBr): $v$ 1710, 1622, $1469 \mathrm{~cm}^{-1} .{ }^{1} \mathrm{H}-\mathrm{NMR}\left(400 \mathrm{MHz}, \mathrm{CDCl}_{3}\right): \delta 7.50(\mathrm{~d}, J=8.0 \mathrm{~Hz}, 1 \mathrm{H}, \mathrm{ArH}), 7.29(\mathrm{t}, J=8.0 \mathrm{~Hz}, 1 \mathrm{H}$, $\operatorname{ArH}), 7.22(\mathrm{~d}, J=8.0 \mathrm{~Hz}, 1 \mathrm{H}, \mathrm{ArH}), 7.18(\mathrm{t}, J=8.0 \mathrm{~Hz}, 1 \mathrm{H}, \mathrm{ArH}), 5.21(\mathrm{ddt}, J=16.8,10.0,6.8 \mathrm{~Hz}, 1 \mathrm{H}$, vinyl $\mathrm{H}), 4.91(\mathrm{~d}, J=16.8 \mathrm{~Hz}, 1 \mathrm{H}$, vinyl $\mathrm{H}), 4.85(\mathrm{~d}, J=10.0 \mathrm{~Hz}, 1 \mathrm{H}$, vinyl $\mathrm{H}), 2.92(\mathrm{ddd}, J=13.6,6.0$, $4.0 \mathrm{~Hz}, 1 \mathrm{H}, \mathrm{CH}), 2.56-2.64(\mathrm{~m}, 2 \mathrm{H}), 2.45(\mathrm{dd}, J=13.6,7.6,1 \mathrm{H}, \mathrm{CH}), 1.97-2.09\left(\mathrm{~m}, 2 \mathrm{H}, \mathrm{CH}_{2}\right), 1.70-1.82(\mathrm{~m}$, $\left.2 \mathrm{H}, \mathrm{CH}_{2}\right), 1.54-1.65\left(\mathrm{~m}, 2 \mathrm{H}, \mathrm{CH}_{2}\right), 1.41-1.51(\mathrm{~m}, 1 \mathrm{H}, \mathrm{CH}), 0.67-0.76(\mathrm{~m}, 1 \mathrm{H}, \mathrm{CH}) ;{ }^{13} \mathrm{C}-\mathrm{NMR}(100 \mathrm{MHz}$, $\left.\mathrm{CDCl}_{3}\right): \delta 190.7(\mathrm{C}), 154.6(\mathrm{C}), 143.5(\mathrm{C}), 132.2(\mathrm{CH}), 127.6(\mathrm{CH}), 124.9(\mathrm{CH}), 121.8(\mathrm{CH}) 119.6(\mathrm{CH})$, $117.9\left(\mathrm{CH}_{2}\right), 62.1(\mathrm{C}), 41.5(\mathrm{CH}), 34.9\left(\mathrm{CH}_{2}\right), 31.3\left(\mathrm{CH}_{2}\right), 30.4\left(\mathrm{CH}_{2}\right), 28.5\left(\mathrm{CH}_{2}\right), 24.5\left(\mathrm{CH}_{2}\right)$. EI-MS: $\mathrm{m} / z$ $225\left(\mathrm{M}^{+}\right), 210,196,184,168,156,143,128,115,89,77,63,51$. EI-HRMS calcd. for $\mathrm{C}_{16} \mathrm{H}_{19} \mathrm{~N}$ : 225.1517 . Found: 225.1516.

N-Allyl-2,3-dimethylindole (3e) [63]: yellow oil. IR (KBr): $v$ 1696, 1614, $1452 \mathrm{~cm}^{-1} .{ }^{1} \mathrm{H}-\mathrm{NMR}(400 \mathrm{MHz}$, $\left.\mathrm{CDCl}_{3}\right): \delta 7.49(\mathrm{dd}, J=7.6,1.2 \mathrm{~Hz}, 1 \mathrm{H}, \mathrm{ArH}), 7.20(\mathrm{dd}, J=7.6,1.2 \mathrm{~Hz}, 1 \mathrm{H}, \mathrm{ArH}), 7.12(\mathrm{ddd}, J=7.6,7.2$, $1.2 \mathrm{~Hz}, 1 \mathrm{H}, \mathrm{ArH}), 7.07(\mathrm{ddd}, J=7.6,7.2,1.2 \mathrm{~Hz}, 1 \mathrm{H}, \mathrm{ArH}), 5.92(\mathrm{ddt}, J=17.2,10.4,4.4 \mathrm{~Hz}, 1 \mathrm{H}$, vinyl $\mathrm{H}), 5.09(\mathrm{ddt}, J=10.4,2.0,1.2 \mathrm{~Hz}, 1 \mathrm{H}$, vinyl $\mathrm{H}), 4.82(\mathrm{ddt}, J=17.2,2.0,1.2 \mathrm{~Hz}, 1 \mathrm{H}$, vinyl H), $4.66(\mathrm{dt}$, $\left.J=4.4,2.0 \mathrm{~Hz}, 2 \mathrm{H}, \mathrm{CH}_{2}\right), 2.31\left(\mathrm{~s}, 3 \mathrm{H}, \mathrm{CH}_{3}\right), 2.26\left(\mathrm{~s}, 3 \mathrm{H}, \mathrm{CH}_{3}\right) ;{ }^{13} \mathrm{C}-\mathrm{NMR}\left(100 \mathrm{MHz}, \mathrm{CDCl}_{3}\right): \delta 136.0(\mathrm{C})$, $133.7(\mathrm{CH}), 132.2(\mathrm{C}), 128.5(\mathrm{C}), 120.5(\mathrm{CH}), 118.7(\mathrm{CH}), 117.9(\mathrm{CH}), 115.9\left(\mathrm{CH}_{2}\right), 108.6(\mathrm{CH}), 106.7(\mathrm{C})$, $45.3\left(\mathrm{CH}_{2}\right), 9.9\left(\mathrm{CH}_{3}\right), 8.8\left(\mathrm{CH}_{3}\right)$. EI-MS: $m / z 185\left(\mathrm{M}^{+}\right), 170,158,144,128,115,102,88,77,51$. EI-HRMS calcd. for $\mathrm{C}_{13} \mathrm{H}_{15} \mathrm{~N}$ : 185.1204 . Found: 185.1202 .

3-Allyl-2,3-dimethyl-3H-indole (4e) [16]: yellow oil. IR (KBr): $v$ 1712, 1638, 1604, $1453 \mathrm{~cm}^{-1} .{ }^{1} \mathrm{H}-\mathrm{NMR}$ $\left(400 \mathrm{MHz}, \mathrm{CDCl}_{3}\right): \delta 7.50(\mathrm{dd}, J=7.6,1.2 \mathrm{~Hz}, 1 \mathrm{H}, \mathrm{ArH}), 7.28(\mathrm{dd}, J=7.6,1.2 \mathrm{~Hz}, 1 \mathrm{H}, \mathrm{ArH}), 7.23-7.26$ $(\mathrm{m}, 1 \mathrm{H}, \mathrm{ArH}), 7.16(\mathrm{ddd}, J=7.6,7.2,1.2 \mathrm{~Hz}, 1 \mathrm{H}, \mathrm{ArH}), 5.13(\mathrm{ddt}, J=17.2,10.4,6.4 \mathrm{~Hz}, 1 \mathrm{H}$, vinyl H), $4.92(\mathrm{ddt}, J=17.2,2.0,1.2 \mathrm{~Hz}, 1 \mathrm{H}$, vinyl H), $4.83(\mathrm{ddt}, J=10.0,2.0,1.2 \mathrm{~Hz}, 1 \mathrm{H}$, vinyl H), 2.60 (ddt, $J=13.6,6.4,1.2 \mathrm{~Hz}, 1 \mathrm{H}, \mathrm{CH}), 2.38(\mathrm{ddt}, J=13.6,8.0,1.2 \mathrm{~Hz}, 1 \mathrm{H}, \mathrm{CH}), 2.33\left(\mathrm{~s}, 3 \mathrm{H}, \mathrm{CH}_{3}\right), 1.28(\mathrm{~s}, 3 \mathrm{H}$, $\left.\mathrm{CH}_{3}\right) ;{ }^{13} \mathrm{C}-\mathrm{NMR}\left(100 \mathrm{MHz}, \mathrm{CDCl}_{3}\right): \delta 186.4(\mathrm{C}), 154.1(\mathrm{C}), 143.3(\mathrm{C}), 132.4(\mathrm{CH}), 127.6(\mathrm{CH}), 124.9(\mathrm{CH})$, $121.7(\mathrm{CH}), 119.7(\mathrm{CH}), 117.9\left(\mathrm{CH}_{2}\right), 57.4\left(\mathrm{CH}_{2}\right), 41.1\left(\mathrm{CH}_{2}\right), 21.7\left(\mathrm{CH}_{3}\right), 15.8\left(\mathrm{CH}_{3}\right)$. EI-MS: $m / z 185$ $\left(\mathrm{M}^{+}\right), 170,158,144,128,115,102,88,77,51 . E I-H R M S$ calcd. for $\mathrm{C}_{13} \mathrm{H}_{15} \mathrm{~N}$ : 185.1204. Found: 185.1202.

N-Allyl-3-methylindole (3f) [64]: yellow oil. IR ( $\mathrm{KBr}): v 1614,1459 \mathrm{~cm}^{-1} .{ }^{1} \mathrm{H}-\mathrm{NMR}\left(400 \mathrm{MHz}, \mathrm{CDCl}_{3}\right)$ : $\delta 7.57(\mathrm{ddd}, J=8.4,1.2,0.8 \mathrm{~Hz}, 1 \mathrm{H}, \mathrm{ArH}), 7.27(\mathrm{ddd}, J=8.0,1.2,0.8 \mathrm{~Hz}, 1 \mathrm{H}, \mathrm{ArH}), 7.18(\mathrm{ddd}, J=8.4,6.8$, $1.2 \mathrm{~Hz}, 1 \mathrm{H}, \mathrm{ArH}), 7.10(\mathrm{ddd}, J=8.0,6.8,0.8 \mathrm{~Hz}, 1 \mathrm{H}, \mathrm{ArH}), 6.86(\mathrm{~s}, J=1 \mathrm{~Hz}, \mathrm{ArH}), 5.96(\mathrm{ddt}, J=17.2$, 10.0, $5.2 \mathrm{~Hz}, 1 \mathrm{H}$, vinyl H), $5.16(\mathrm{ddt}, J=10.0,1.6,1.2 \mathrm{~Hz}, 1 \mathrm{H}$, vinyl H), $5.07(\mathrm{ddt}, J=17.2,1.6,1.2 \mathrm{~Hz}, 1 \mathrm{H}$, vinyl $\mathrm{H}), 4.65\left(\mathrm{dt}, J=5.2,1.6 \mathrm{~Hz}, 2 \mathrm{H}, \mathrm{NCH}_{2}\right), 2.32\left(\mathrm{~s}, 3 \mathrm{H}, \mathrm{CH}_{3}\right) ;{ }^{13} \mathrm{C}-\mathrm{NMR}\left(100 \mathrm{MHz}, \mathrm{CDCl}_{3}\right): \delta 136.4$ (C), $133.8(\mathrm{CH}), 128.9(\mathrm{C}), 125.4(\mathrm{CH}), 121.4(\mathrm{CH}), 119.0(\mathrm{CH}), 118.6(\mathrm{CH}), 117.0\left(\mathrm{CH}_{2}\right), 110.5(\mathrm{C}), 109.3$ 
(CH), $48.5\left(\mathrm{CH}_{2}\right), 9.6\left(\mathrm{CH}_{3}\right)$. EI-MS: $m / z 171\left(\mathrm{M}^{+}\right), 156,144,130,129,103,89,77,51$. EI-HRMS calcd. for $\mathrm{C}_{12} \mathrm{H}_{13} \mathrm{~N}$ : 171.1048. Found: 171.1051.

3-Allyl-3-methyl-3H-indole (4f) [16]: yellow oil. IR (KBr): $v$ 1711, 1638, 1603, $1479 \mathrm{~cm}^{-1} .{ }^{1} \mathrm{H}-\mathrm{NMR}$ $\left(400 \mathrm{MHz}, \mathrm{CDCl}_{3}\right): \delta 8.03(\mathrm{~s}, 1 \mathrm{H}, \mathrm{CH}=\mathrm{N}), 7.63(\mathrm{dd}, J=7.6,1.2 \mathrm{~Hz}, 1 \mathrm{H}, \mathrm{ArH}), 7.34(\mathrm{dt}, J=7.6,1.2 \mathrm{~Hz}, 1 \mathrm{H}$, ArH), $7.31(\mathrm{dd}, J=7.2,1.2 \mathrm{~Hz}, 1 \mathrm{H}, \mathrm{ArH}), 7.26(\mathrm{ddd}, J=7.6,7.2,1.2 \mathrm{~Hz}, 1 \mathrm{H}, \mathrm{ArH}), 5.51(\mathrm{ddt}, J=17.2$, 10.0, 7.2 Hz, 1H, vinyl H), $5.02(\mathrm{ddt}, J=17.2,2.0,1.2 \mathrm{~Hz}, 1 \mathrm{H}$, vinyl $\mathrm{H}), 4.98(\mathrm{ddt}, J=10.0,2.0,1.2 \mathrm{~Hz}, 1 \mathrm{H}$, vinyl $\mathrm{H}), 2.49(\mathrm{dt}, J=7.2,1.2 \mathrm{~Hz}, 1 \mathrm{H}, \mathrm{CH}), 2.48(\mathrm{dt}, J=7.2,1.2 \mathrm{~Hz}, 1 \mathrm{H}, \mathrm{CH}), 1.35\left(\mathrm{~s}, 3 \mathrm{H}, \mathrm{CH}_{3}\right) ;{ }^{13} \mathrm{C}-\mathrm{NMR}$ $\left(100 \mathrm{MHz}, \mathrm{CDCl}_{3}\right): \delta 178.9(\mathrm{CH}), 154.8(\mathrm{C}), 143.2(\mathrm{C}), 132.8(\mathrm{CH}), 127.8(\mathrm{CH}), 126.1(\mathrm{CH}), 121.7(\mathrm{CH})$, 121.2 (CH), $118.6\left(\mathrm{CH}_{2}\right), 57.0(\mathrm{C}), 40.2\left(\mathrm{CH}_{2}\right), 19.6\left(\mathrm{CH}_{3}\right)$. EI-MS: $m / z 170\left(\mathrm{M}^{+}\right), 156,144,128,115,103$, 77, 51. EI-HRMS calcd. for $\mathrm{C}_{12} \mathrm{H}_{13} \mathrm{~N}$ : 171.1048. Found: 171.1047 .

\section{Conclusions}

In conclusion, we developed a catalytic system that used platinum-catalyzed allylation of heterocycle fused indoles with allylic acetates for a convenient and simple method to form C-N or $\mathrm{C}-\mathrm{C}$ bonds. The reaction condition did not occur without any platinum catalyst and phosphine ligand. The allylation of allylic acetates worked well with carbazoles, giving generally good yields of corresponding allylic carbazoles.

Acknowledgments: This work was supported by a grant from National Science Councial of Republic of China (NSC101-2113-M-037-008-MY3).

Author Contributions: Shyh-Chyun Yang conceived and designed the experiments; Bai-Jing Peng and Wen-Ting Wu performed the experiments; Bai-Jing Peng and Shyh-Chyun Yang analyzed the data; Shyh-Chyun Yang contributed reagents/materials/analysis tools; Bai-Jing Peng and Shyh-Chyun Yang wrote the paper.

Conflicts of Interest: The authors declare no conflict of interest.

\section{References}

1. Gul, W.; Hamann, M.T. Indole alkaloid marine natural products: An established source of cancer drug leads with considerable promise for the control of parasitic, neurological and other diseases. Life Sci. 2005, 78, 442-453. [CrossRef] [PubMed]

2. Kawasaki, T.; Higuchi, K. Simple indole alkaloids and those with a nonrearranged monoterpenoid unit. Nat. Prod. Rep. 2005, 22, 761-793. [CrossRef] [PubMed]

3. Kochanowska-Karamyan, A.J.; Hamann, M.T. Marine indole alkaloids: Potential new drug leads for the control of depression and anxiety. Chem. Rev. 2010, 110, 4489-4497. [CrossRef] [PubMed]

4. Maincent, P.; Le Verge, R.; Sado, P.; Couvreur, P.; Devissaguet, J.P. Disposition kinetics and oral bioavailability of vincamine-loaded polyalkyl cyanoacrylate nanoparticles. J. Pharm. Sci. 1986, 75, 955-958. [CrossRef] [PubMed]

5. Rahbæk, L.; Christophersen, C. Marine alkaloids. 19. Three new alkaloids, securamines e-g, from the marine bryozoan securiflustra securifrons. J. Nat. Prod. 1997, 60, 175-177. [CrossRef]

6. Vepsäläinen, J.J.; Auriola, S.; Tukiainen, M.; Ropponen, N.; Callaway, J.C. Isolation and characterization of yuremamine, a new phytoindole. Planta Med. 2005, 71, 1053-1057. [CrossRef] [PubMed]

7. Fernandez, L.S.; Buchanan, M.S.; Carroll, A.R.; Feng, Y.J.; Quinn, R.J.; Avery, V.M. Flinderoles a-c: Antimalarial bis-indole alkaloids from flindersia species. Org. Lett. 2009, 11, 329-332. [CrossRef] [PubMed]

8. Vallakati, R.; Smuts, J.P.; Armstrong, D.W.; May, J.A. On the biosynthesis and optical activity of the flinderoles. Tetrahedron Lett. 2013, 54, 5892-5894. [CrossRef]

9. Kimura, M.; Futamata, M.; Mukai, R.; Tamaru, Y. Pd-catalyzed c3-selective allylation of indoles with allyl alcohols promoted by triethylborane. J. Am. Chem. Soc. 2005, 127, 4592-4593. [CrossRef] [PubMed]

10. Joule, J.A.; Mills, K. Heterocyclic Chemistry; John Wiley \& Sons: Hoboken, NJ, USA, 2008.

11. Sundberg, R.J. Indoles; Academic Press: Cambridge, MA, USA, 1996.

12. Bandini, M.; Melloni, A.; Tommasi, S.; Umani-Ronchi, A. A journey across recent advances in catalytic and stereoselective alkylation of indoles. Synlett 2005, 2005, 1199-1222. [CrossRef] 
13. Cacchi, S.; Fabrizi, G. Synthesis and functionalization of indoles through palladium-catalyzed reactions. Chem. Rev. 2005, 105, 2873-2920. [CrossRef] [PubMed]

14. Lakhdar, S.; Westermaier, M.; Terrier, F.; Goumont, R.; Boubaker, T.; Ofial, A.R.; Mayr, H. Nucleophilic reactivities of indoles. J. Org. Chem. 2006, 71, 9088-9095. [CrossRef] [PubMed]

15. Otero, N.; Mandado, M.; Mosquera, R.A. Nucleophilicity of indole derivatives: Activating and deactivating effects based on proton affinities and electron density properties. J. Phys. Chem. A 2007, 111, 5557-5562. [CrossRef] [PubMed]

16. Kagawa, N.; Malerich, J.P.; Rawal, V.H. Palladium-catalyzed $\beta$-allylation of 2,3-disubstituted indoles. Org. Lett. 2008, 10, 2381-2384. [CrossRef] [PubMed]

17. Li, C.; Chan, C.; Heimann, A.C.; Danishefsky, S.J. On the rearrangement of an azaspiroindolenine to a precursor to phalarine: Mechanistic insights. Angew. Chem. Int. Ed. 2007, 46, 1444-1447. [CrossRef] [PubMed]

18. Sapi, J.; Dridi, S.; Laronze, J.; Sigaut, F.; Patigny, D.; Laronze, J.-Y.; Lévy, J.; Toupet, L. Indole as a tool in synthesis. Indolenine approach to 4,5-epoxy-10-normorphinans. Tetrahedron 1996, 52, 8209-8222. [CrossRef]

19. Vercauteren, J.; Massiot, G.; Levy, J. Methyleneindolines, indolenines, and indoleniniums. 19. A new entry into the hexahydropyrrolidino[2,3-d]carbazole system. J. Org. Chem. 1984, 49, 3230-3231. [CrossRef]

20. Bramely, R.K.; Caldwell, J.; Grigg, R. Site specificity of [3,3] sigmatropic rearrangements of 3-allyl- and 3-(prop-2-ynyl)-3h-indoles. J. Chem. Soc. Perkin Trans. 1 1973, 17, 1913-1921. [CrossRef] [PubMed]

21. Robinson, R.; Suginome, H. 41. Experiments on the synthesis of physostigmine (eserine). Part i. Some indolenine derivatives. J. Chem. Soc. (Resumed) 1932, 298-304. [CrossRef]

22. Zaitsev, A.B.; Gruber, S.; Plüss, P.A.; Pregosin, P.S.; Veiros, L.F.; Wörle, M. Fast and highly regioselective allylation of indole and pyrrole compounds by allyl alcohols using ru-sulfonate catalysts. J. Am. Chem. Soc. 2008, 130, 11604-11605. [CrossRef] [PubMed]

23. Liu, W.-B.; He, H.; Dai, L.-X.; You, S.-L. Ir-catalyzed regio- and enantioselective friedel-crafts-type allylic alkylation of indoles. Org. Lett. 2008, 10, 1815-1818. [CrossRef] [PubMed]

24. Sundararaju, B.; Achard, M.; Demerseman, B.; Toupet, L.; Sharma, G.V.M.; Bruneau, C. Ruthenium(iv) complexes featuring p,o-chelating ligands: Regioselective substitution directly from allylic alcohols. Angew. Chem. Int. Ed. 2010, 49, 2782-2785. [CrossRef] [PubMed]

25. Jiao, L.; Herdtweck, E.; Bach, T. Pd(II)-catalyzed regioselective 2-alkylation of indoles via a norbornene-mediated C-H activation: Mechanism and applications. J. Am. Chem. Soc. 2012, 134, 14563-14572. [CrossRef] [PubMed]

26. Weaver, J.D.; Recio, A.; Grenning, A.J.; Tunge, J.A. Transition metal-catalyzed decarboxylative allylation and benzylation reactions. Chem. Rev. 2011, 111, 1846-1913. [CrossRef] [PubMed]

27. Ikeda, M.; Miyake, Y.; Nishibayashi, Y. Cooperative catalytic reactions using organocatalysts and transition metal catalysts: Propargylic allylation of propargylic alcohols with $\alpha, \beta$-unsaturated aldehydes. Organometallics 2012, 31, 3810-3813. [CrossRef]

28. Schwarz, K.J.; Amos, J.L.; Klein, J.C.; Do, D.T.; Snaddon, T.N. Uniting C1-ammonium enolates and transition metal electrophiles via cooperative catalysis: The direct asymmetric $\alpha$-allylation of aryl acetic acid esters. J. Am. Chem. Soc. 2016, 138, 5214-5217. [CrossRef] [PubMed]

29. Kumar, D.; Vemula, S.R.; Balasubramanian, N.; Cook, G.R. Indium-mediated stereoselective allylation. Acc. Chem. Res. 2016, 49, 2169-2178. [CrossRef] [PubMed]

30. Finke, R. Principles and Applications of Organotransition Metal Chemistry; University Science Book: Mill Valley, CA, USA, 1987.

31. Balasubramanian, N.; Mandal, T.; Cook, G.R. Highly diastereoselective palladium-catalyzed indium-mediated allylation of chiral hydrazones. Org. Lett. 2015, 17, 314-317. [CrossRef] [PubMed]

32. Huang, W.-Y.; Nishikawa, T.; Nakazaki, A. Palladium-catalyzed cascade wacker/allylation sequence with allylic alcohols leading to allylated dihydropyrones. ACS Omega 2017, 2, 487-495. [CrossRef]

33. Shimizu, M.; Kimura, M.; Watanabe, T.; Tamaru, Y. Palladium-catalyzed allylation of imines with allyl alcohols. Org. Lett. 2005, 7, 637-640. [CrossRef] [PubMed]

34. Montgomery, T.D.; Zhu, Y.; Kagawa, N.; Rawal, V.H. Palladium-catalyzed decarboxylative allylation and benzylation of n-alloc and n-cbz indoles. Org. Lett. 2013, 15, 1140-1143. [CrossRef] [PubMed]

35. Hikawa, H.; Yokoyama, Y. Palladium-catalyzed mono- $n$-allylation of unprotected anthranilic acids with allylic alcohols in aqueous media. J. Org. Chem. 2011, 76, 8433-8439. [CrossRef] [PubMed]

36. Hossian, A.; Singha, S.; Jana, R. Palladium(0)-catalyzed intramolecular decarboxylative allylation of ortho nitrobenzoic esters. Org. Lett. 2014, 16, 3934-3937. [CrossRef] [PubMed] 
37. Tsuji, J. Palladium Reagents and Catalysts; Wiley \& Sons: Hoboken, NJ, USA, 1995.

38. Trost, B.M. Cyclizations via palladium-catalyzed allylic alkylations [new synthetic methods (79)]. Angew. Chem. Int. Ed. Engl. 1989, 28, 1173-1192. [CrossRef]

39. Oppolzer, $\mathrm{W}$. Intramolecular, stoichiometric $(\mathrm{Li}, \mathrm{Mg}, \mathrm{Zn})$ and catalytic $(\mathrm{Ni}, \mathrm{Pd}, \mathrm{Pt})$ metallo-ene reactions in organic synthesis [new synthetic methods (75)]. Angew. Chem. Int. Ed. Engl. 1989, 28, 38-52. [CrossRef]

40. Tsuji, J. Expanding industrial applications of palladium catalysts. Synthesis 1990, 1990, 739-749. [CrossRef]

41. Uozumi, Y.; Danjo, H.; Hayashi, T. Cross-coupling of aryl halides and allyl acetates with arylboron reagents in water using an amphiphilic resin-supported palladium catalyst. J. Org. Chem. 1999, 64, 3384-3388. [CrossRef] [PubMed]

42. Rajesh, S.; Banerji, B.; Iqbal, J. Palladium(0)-catalyzed regioselective synthesis of $\alpha$-dehydro- $\beta$-amino esters from amines and allyl acetates: Synthesis of a $\alpha$-dehydro- $\beta$-amino acid derived cyclic peptide as a constrained $\beta$-turn mimic. J. Org. Chem. 2002, 67, 7852-7857. [CrossRef] [PubMed]

43. Wallner, O.A.; Szabó, K.J. Palladium-catalyzed electrophilic substitution of allyl chlorides and acetates via bis-allylpalladium intermediates. J. Org. Chem. 2003, 68, 2934-2943. [CrossRef] [PubMed]

44. Deardorff, D.R.; Savin, K.A.; Justman, C.J.; Karanjawala, Z.E.; Sheppeck, J.E.; Hager, D.C.; Aydin, N. Conversion of allylic alcohols into allylic nitromethyl compounds via a palladium-catalyzed solvolysis: An enantioselective synthesis of an advanced carbocyclic nucleoside precursor1. J. Org. Chem. 1996, 61, 3616-3622. [CrossRef] [PubMed]

45. Kadota, J.; Katsuragi, H.; Fukumoto, Y.; Murai, S. Platinum and palladium complex-catalyzed regioselective nucleophilic substitutions with two different nucleophiles at the central and terminal carbon atoms of the $\pi$-allyl ligand. Organomet. 2000, 19, 979-983. [CrossRef]

46. Kamijo, S.; Jin, T.; Yamamoto, Y. Novel synthetic route to allyl cyanamides: Palladium-catalyzed coupling of isocyanides, allyl carbonate, and trimethylsilyl azide. J. Am. Chem. Soc. 2001, 123, 9453-9454. [CrossRef] [PubMed]

47. Minami, I.; Yuhara, M.; Tsuji, J. 1-Isopropylallyloxycarbonyl (ipaoc) as a protective group of amines and its deprotection catalysed by palladium-phosphine complex. Tetrahedron Lett. 1987, 28, 2737-2740. [CrossRef]

48. Kawabata, T.; Itoh, K.; Hiyama, T. Regio- and chemoselective epimerization of cis-3-amino- $\beta$-lactams to the trans-isomers: A new synthesis of aztreonam. Tetrahedron Lett. 1989, 30, 4837-4840. [CrossRef]

49. Ziegler, F.E.; Wester, R.T. Regiochemical control in the hemiacetalization of a dihydroxydialdehyde. An application of the use of homochiral 3-methyl- $\delta$-butyrolactones to the construction of homochiral tripropionate units. Tetrahedron Lett. 1986, 27, 1225-1228. [CrossRef]

50. Ziegler, F.E.; Cain, W.T.; Kneisley, A.; Stirchak, E.P.; Wester, R.T. Applications of the 3-methyl-.Gamma.-butyrolactone strategy to the synthesis of polypropionates: The prelog-djerassi lactonic ester, ent-invictolide, and the c19-c27 fragment of rifamycin s. J. Am. Chem. Soc. 1988, 110, 5442-5452. [CrossRef]

51. Schenck, T.G.; Bosnich, B. Homogeneous catalysis. Transition-metal-catalyzed claisen rearrangements. J. Am. Chem. Soc. 1985, 107, 2058-2066. [CrossRef]

52. Auburn, P.R.; Whelan, J.; Bosnich, B. Homogeneous catalysis. Production of allyl alkyl sulphides by palladium mediated allylation. J. Chem. Soc. Chem. Commun. 1986, 17, 146-147. [CrossRef]

53. Tamura, R.; Kamimura, A.; Ono, N. Displacement of aliphatic nitro groups by carbon and heteroatom nucleophiles. Synthesis 1991, 1991, 423-434. [CrossRef]

54. Trost, B.M.; Schmuff, N.R.; Miller, M.J. Allyl sulfones as synthons for 1,1- and 1,3-dipoles via organopalladium chemistry. J. Am. Chem. Soc. 1980, 102, 5979-5981. [CrossRef]

55. Yasushi, T.; Jun, S.; Ryo, T.; Yoshihisa, W. The platinum complex catalyzed transformation of primary amine to secondary amine. Chem. Lett. 1984, 13, 889-890.

56. Yasushi, T.; Ryo, T.; Hiroshi, O.; Yoshihisa, W. Platinum complex catalyzed transformation of amine. $\mathrm{N}$-alkylation and N-allylation using primary alcohols. Chem. Lett. 1986, 15, 293-294.

57. Yang, S.-C.; Tsai, Y.-C.; Shue, Y.-J. Direct platinum-catalyzed allylation of anilines using allylic alcohols. Organometallics 2001, 20, 5326-5330. [CrossRef]

58. Huynh, K.Q.; Seizert, C.A.; Ozumerzifon, T.J.; Allegretti, P.A.; Ferreira, E.M. Platinum-catalyzed $\alpha, \beta$-unsaturated carbene formation in the formal syntheses of frondosin b and liphagal. Org. Lett. 2017, 19, 294-297. [CrossRef] [PubMed]

59. Labinger, J.A. Platinum-catalyzed C-H functionalization. Chem. Rev. 2017, 117, 8483-8496. [CrossRef] [PubMed] 
60. Gan, K.-H.; Jhong, C.-J.; Shue, Y.-J.; Yang, S.-C. Platinum-catalyzed allylation of aminonaphthalenes with allylic acetates in water. Tetrahedron 2008, 64, 9625-9629. [CrossRef]

61. Yang, S.-C.; Feng, W.-H.; Gan, K.-H. Platinum-catalyzed allylation of aminonaphthalenes with allylic acetates. Tetrahedron 2006, 62, 3752-3760. [CrossRef]

62. Zhang, X.; Yang, Z.-P.; Liu, C.; You, S.-L. Ru-catalyzed intermolecular dearomatization reaction of indoles with allylic alcohols. Chem. Sci. 2013, 4, 3239-3243. [CrossRef]

63. Motoki, Y.; Yuko, K.; Koichi, N. Palladium-catalyzed carboacylation of alkenes by using acylchromates as acyl donors. Bull. Chem. Soc. Jpn. 2005, 78, 331-340.

64. Masao, N. Alkylation of 2,3-dimethylindole in liquid ammonia. Bull. Chem. Soc. Jpn. 1959, 32, 838-840.

Sample Availability: Samples of the compounds $3 a, 4 a, 3 b, 4 b, 3 c, 4 c, 3 d, 4 d, 3 e, 4 e, 3 f$, and $4 f$ are available from the authors.

(C) 2017 by the authors. Licensee MDPI, Basel, Switzerland. This article is an open access article distributed under the terms and conditions of the Creative Commons Attribution (CC BY) license (http:// creativecommons.org/licenses/by/4.0/). 